\title{
Regulation of Gastric Acid Secretion of Liriope platyphylla Extract in Gastroesophageal Reflux Disease
}

\author{
Sang Hyun $\mathrm{Ahn}^{1}$, Il Shin $\mathrm{Choi}^{2}$, Ki Bong $\mathrm{Kim}^{2,3^{*}}$ \\ ${ }^{1}$ Department of Anatomy, College of Korean Medicine, Semyung University \\ ${ }^{2}$ Department of Korean Pediatrics, Pusan National University Korean Hospital \\ ${ }^{3}$ School of Korean Medicine, Pusan National University
}

\begin{abstract}
Objectives: The purpose of this study was to confirm the effects of Liriope platyphylla extract on relieving Gastroesophageal reflux disease (GERD) through regulation of acid secretion.

Methods: 8-week-old ICR mice were divided into untreated control group (Ctrl), GERD elecitation group (GERDE), Omeprazole administrate group before GERD elicitation (OMA), and Liriope platyphylla extract administrate group before GERD elicitation (LPA). After inducing GERD, gross observation and histological examination were performed and ATP6V1B1 (ATPase H+ Transporting V1 Subunit B1), GRPR (Gastrin-releasing peptide receptor), COX-1 (Cyclooxygenase 1), 8-OHdG (8-hydroxy-2'-deoxyguanosine), Cathelicidin, p-JNK (phospho c-Jun N-terminal kinase) were observed to confirm the damage defense effect of the esophageal mucosa, acid secretion regulation, antioxidant, anti-inflammatory, mucosal protection, and apoptosis regulation

Results: OMA and LPA showed lower levels of damage compared to GERDE in gross observation and histological examination. ATP6V1B1, GRPR, and 8-OHdG showed lower positive reactions in OMA and LPA than in GERDE. COX-1 were less positive in GERDE and OMA than in Ctrl, but showed higher secretion in LPA than in Ctrl. Cathelicidin showed a decreased positive reaction in GERDE, OMA and LPA compared to Ctrl, but the decrease in positive reaction was smaller in OMA and LPA compared to GERDE. p-JNK showed increased positive reaction in GERDE, OMA and LPA than in Ctrl, but the increase in the positive reaction was smaller in the OMA and LPA compared to GERDE.

Conclusions: The effects of Liriope platyphylla extract on esophageal mucosal damage protection, acid secretion regulation, antioxidant, anti-inflammatory, mucosal protection and apoptosis regulation were confirmed.
\end{abstract}

$\overline{K e y}$ Words : Liriope platyphylla, Gastroesophageal reflux disease, acid secretion regulation, Cathelicidin, $\mathrm{p}-\mathrm{JNK}$

\section{Introduction}

Gastroesophageal reflux disease (GERD) occurs when small amounts of stomach contents reflux into the esophagus, and this reflux phenomenon continues. It mainly causes symptoms such as heartburn, acid reflux, difficulty swallowing, dysphagia, nausea, and chronic cough ${ }^{1)}$. This is caused by an abnormality in the sphincter that prevents the reflux of food between the stomach and the esophagus. If the damage to the esophageal mucosa persists, reflux esophagitis

- Received : 29 September 2021

- Revised : 25 October 2021

- Accepted : 5 November 2021

- Correspondence to : Kibong Kim

Department of Korean Pediatrics, Pusan National University Korean Hospital

Geumo-ro 20, Mulgeum-eup, Yangsan-si, Gyeongsangnam-do, 50612, Republic of Korea

Tel : +82-55-360-5952, Fax : +82-55-360-5952, E-mail : kkb@pusan.ac.kr 
occurs, and when it progresses to erosive esophagitis, the mucosal damage becomes more evident. If reflux esophagitis persists for a long time, esophageal stricture may occur and as esophagitis heals, cellular dysplasia may be induced, resulting in Barrett's esophagus. If the dysplasia of cells becomes severe, it may progress to esophageal or stomach cancer. Although the incidence of esophageal cancer isn't relatively high, the mortality rate is very high and the prognosis is poor, so the 5-year survival rate is only less than $5 \%{ }^{2)}$. Therefore, if GERD is not actively treated, it may increase the potential risk factors for cancer in the long term.

Currently, the representative treatment for GERD is proton pump inhibitors (PPIs) ${ }^{1)}$. PPI binds to and inactivates the proton pump of gastric parietal cells, thereby inhibiting acid secretion, which is now used as a treatment for GERD ${ }^{3)}$. However, when accompanied by esophagitis and Barrett's esophagus, PPI is used to suppress acid secretion, but this inhibition of acid secretion does not fundamentally cure or prevent esophagitis or Barrett's esophagus ${ }^{2)}$. In addition, various side effects such as increased likelihood of cardiovascular -related symptoms due to hypomagnesemia, memory impairment and neurological side effects, and the risk of toxicity due to interaction with other drugs have been reported, and the proportion of domestic refractory GERD that does not respond to drugs is approximately $26 \%{ }^{4-6)}$. Moreover, the use of PPIs increases the risk of osteoporotic fractures, which is pointed out as a problem in an aging society ${ }^{7}$. For this reason, research on drugs that can replace PPI by using a method different from the existing PPI mechanism is required.

Liriope platyphylla (麥門冬) is the tuber of Liriope platyphalla Wang et Tang, a perennial plant belonging to the family Liliaceae, collected in summer and dried. Liriope platyphylla is slightly cold-natured (微寒), sweet but slightly bitter flavored (甘微苦) herb. It acts on lung, stomach and heart channel (肺胃心經) and has nourishing Eum and moistening the lung (養陰潤 肺), clearing away the heart-fire and relieving restlessness (清心除煩), reinforcing the stomach and promoting production of body fluid (益胃生 津) effects. It is used to treat lung dryness-cough (肺燥咳嗽), Eum deficiency-cough (陰虛咳嗽), irritability and sleeplessness (心煩失眠), hematemesis (吐血), consumptive lung disease (肺瘘), lung abscess (肺癱), dysphoria with smothery sensation caused by consumptive disease (虛勞煩熱), emaciation-thirst (消渴), dry throat and mouth (咽 乾口燥), constipation $(\text { 便祕 })^{8)}$. In particular, the oriental medicinal efficacy of Liriope platyphalla, which is clearing away the heart-fire and relieving restlessness (清心除煩), reinforcing the stomach and promoting production of body fluid (分胃生津), is thought to be applicable to the typical symptoms of GERD, such as hearburn and atypical chest pain. In addition, various studies related to Liriope platyphylla have reported anti-inflammatory and antioxidant effects ${ }^{9-12}$. Therefore, based on the anti-inflammatory and antioxidant effects reported in previous studies, Liriope platyphylla would be effective in the treatment and prevention of reflux esophagitis.

Concerning GERD and reflux esophagitis, there were studies on a variety of prescriptions and single or complex medications such as 
Banhasasim-tang $^{13)}$, Yijin-tang ${ }^{14,15)}$, Jwakum-whan ${ }^{16)}$, Coptis Chinensis $^{17)}$, Artemisia Capillaris Herba ${ }^{18)}$, Evodiae Fructus $^{19,20)}$, Citri Pericarpium ${ }^{21)}$, and Ulmus Pumila Cortex ${ }^{22)}$. However, there was no study comparing the therapeutic effect of Liriope platyphylla with PPI in studies related to GERD and reflux esophagitis.

In this study, using an animal model of gastroesophageal reflux disease, the effect of Liriope platyphylla extract on the inhibition of esophageal mucosal damage was visually observed and immunohistochemical tests were performed to evaluate antioxidant, anti-inflammatory, mucosal protection, apoptosis regulation, acid secretion regulation, and visceral nervous system regulation effect of it. Through this, the inhibitory effect of Liriope platyphylla on the development of reflux esophagitis was confirmed by comparing it with Omeprazole.

\section{Materials and Methods}

\section{Materials}

\section{1) Animals}

8-week-old ICR male mice (Junga Bio, Korea) were acclimatized for 2 weeks in an aseptic breeding apparatus, and then mice weighing $30 \pm$ $2 \mathrm{~g}$ were selected. They were divided into Untreated control group (Ctrl), Gastroesophageal reflux disease elecitation group (GERDE), Omeprazole administrate group before GERD elicitation (OMA), Liriope platyphylla extract administrate group before GERD elicitation (LPA) and 10 mice were assigned to each group. A high-fat diet (fat $60 \%$, carbohydrate $20 \%$, protein $20 \%$; DIO DIET, USA) was ad libitum to increase the frequency of reflux symptoms during the duration of the experiment ${ }^{23)}$. This research process was conducted with the approval of Semyung University IACUC (IACUC number: smecae 20-09-02), and was conducted according to the NIH guidelines for the care and use of laboratory animals.

\section{2) Experimental drug}

Liriope platyphylla (100 g, Omniherb, Korea) was added to $1000 \mathrm{ml}$ of distilled water, boiled for 3 hours, and then filtered. The filtrate was decompressed and concentrated to $50 \mathrm{ml}$ using a rotary evaporator, and then freeze-dried to extract 24.5 g (yield: $24.5 \%$ ). After diluting the obtained Liriope platyphylla extract in physiological saline at an amount of $40 \mathrm{mg} / \mathrm{kg}$ daily from 14 days before GERD induction, $100 \mu \mathrm{l}$ was orally administered, and the last drug administration was performed 1 hour before surgery.

Omeprazole (Shinil Pharmaceutical, Korea), a proton pump inhibitor, was used as a control drug, and $40 \mathrm{mg} / \mathrm{kg}$ was orally administered daily from 14 days before GERD induction.

\section{Methods}

1) Reflux esophagitis induction

Reflux esophagitis was induced using the method of Nakamura et $\mathrm{al}^{24)}$. After anesthetizing the rats, which had been fasted for 24 hours, with sodium pentobarbital, the abdominal cavity was opened, and the base and cardiac region of the stomach were ligated with a micro-cable tie (Daihan Science, Korea). After 48 hours of suture, the gastroesophageal junction (GEJ) of the experimental animal was cut off and used in the 
experiment. In addition, to observe changes in the external shape around the gastroesophageal junction, images were taken at $4 \mathrm{x}$ magnification using a steroscope (Olympus, Japan).

\section{2) Tissue section production}

Cardiac perfusion was fixed with vascular rinse and $10 \%$ neutral buffered formalin (NBF). The gastroesophageal junction and lower esophagus were excised and fixed with $10 \% \mathrm{NBF}$ at room temperature for 24 hours. After the fixed tissue was embedded in paraffin, it was prepared as a continuous section with a thickness of $5 \mu \mathrm{m}$.

\section{3) Histochemical test}

A phloxine-tartrazine staining method was performed to observe tissue damage to the gastric mucosa and lower esophagus at the gastroesophageal junction. After nuclear staining with Mayer's hematoxylin for 5 minutes, it was reacted with phloxine solution for 30 minutes. After fractionation from the tartrazine solution, it was observed using an optical microscope (BX 60, Olympus, Japan).

\section{4) Immunohistochemical test}

To investigate immunohistochemical changes in the gastric mucosa of the gastroesophageal junction and the tissues of the lower esophagus, immunohistochemical staining using ATP6V1B1 (ATPase $\mathrm{H}^{+}$Transporting V1 Subunit B1) (Abcam, USA), GRPR (Gastrin-releasing peptide receptor) (Abcam, USA), 8-OHdG (8-hydroxy-2' -deoxyguanosine) (Abcam, USA), COX-1 (Cyclooxygenase-1) (Abcam, USA), Cathelicidin (Abcam, USA) and p-JNK (phospho c-Jun
N-terminal kinase) (Abcam, USA) was performed. The tissue sections were subjected to proteolysis in proteinase $\mathrm{K}(20 \mu \mathrm{g} / \mathrm{ml})$ for 5 minutes, and then reacted in $10 \%$ normal goat serum, a blocking serum, for 2 hours. After reacting the primary antibody in a humidified chamber at $4{ }^{\circ} \mathrm{C}$ for 72 hours, the secondary antibody, biotinylated goat anti-mouse IgG (1:100, Abcam, USA), was linked at room temperature for 24 hours and reacted with avidin biotin complex kit (Vector Lab, USA) for 1 hour at room temperature. After color development in $0.05 \mathrm{M}$ tris- $\mathrm{HCl}$ buffer $(\mathrm{pH}$ 7.4) containing $0.05 \% 3,3$ '-diaminobenzidine and $0.01 \% \mathrm{HCl}$, counterstaining was performed with hematoxylin.

\section{5) Image analysis}

Image analysis was quantified (means \pm standard error) using Image Pro (Media cybernetics, USA). After magnifying the gastroesophageal connection and lower esophageal periphery by 100 times, the intensity (80-100) was measured and counted in the 20,000,000 pixel cell area.

\section{6) Statistics}

For statistics, SPSS software (SPSS 25, SPSS Inc., USA) was used, and one-way ANOVA analysis was performed. Significance was set when the $\mathrm{P}$ value was less than 0.05 , and Tukey HSD was performed for post-test verification.

\section{Results}

\section{Inhibition of gastroesophageal mucosal damage}

After gastric ligation, severe hemorrhagic erosion 


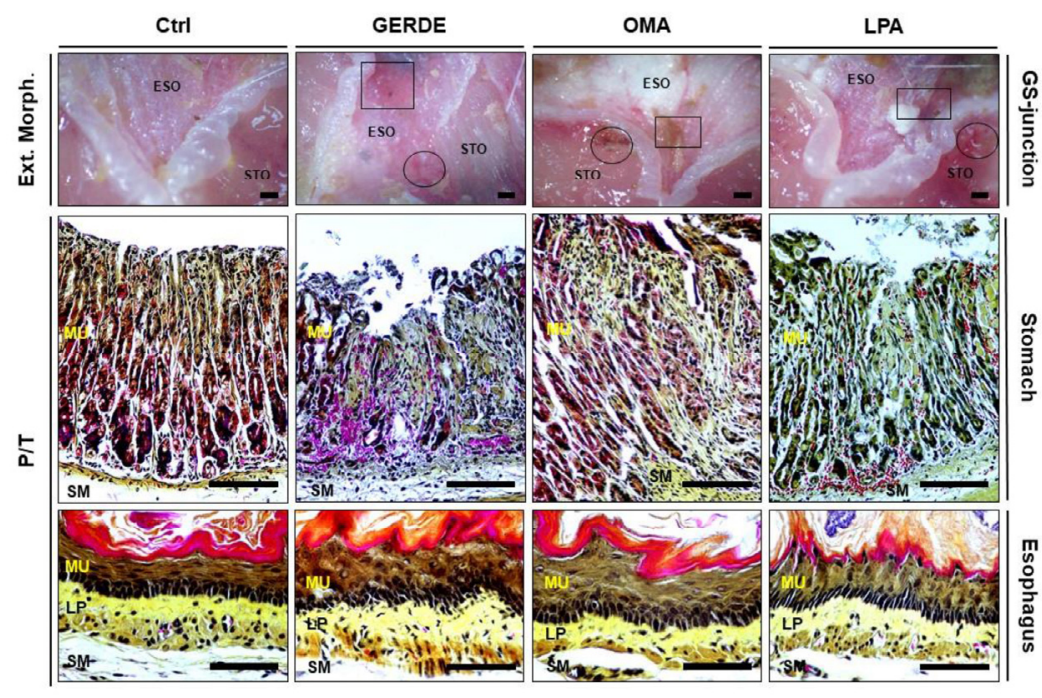

Fig. 1. The alleviation of Gastroesophageal reflux disease (GERD) induced mucosal damages by Omeprazole and Liriope platyphylla.

Gross observation of gastroesophageal junction was performed and histological examination of stomach \& esophagus abrasion site through phloxine-tartrazine staining was observed. A low distribution of hemorrhagic abrasions was observed in OMA and LPA. Histological examination through phloxine-tartrazine staining, severe mucosal damage was observed at the abrasion site. In the order of LPA, OMA, and GERDE, the injury site increased both in stomach \& esophagus. GERD, Gastroesophageal reflux disease; Ctrl, no GERD elicited group; GERDE, GERD elicited group; OMA, Medicine as Omeprazole administrate group before GERD elicitation; LPA, Medicine as extraction of Liriope platyphylla administrate group before GERD elicitation; Circle, hemorrhagic erosion in stomach; Square Box, erosionin esophagus; ESO, Esophagus; STO, Stomach; MU, Mucosa; LP, Laminar propria; SM, submucosa; bar size, 50um.

was observed in the gastric mucosa around the gastroesophageal junction of GERDE. In contrast, a low distribution of hemorrhagic abrasions was observed in OMA and LPA. The degree of damage increased in the order of LPA, OMA, and GERDE (Fig. 1. GS-junction). As a result of histological examination through phloxine-tartrazine staining, severe mucosal damage was observed at the abrasion site. In the order of LPA, OMA, and GERDE, the injury site increased (Fig. 1. Stomach).

After gastric ligation, erosion was observed in the esophageal mucosa around the continuation of the gastroesophageal junction of GERDE. A low distribution of abrasion was observed in
OMA and LPA. The degree of damage increased in the order of LPA, OMA, and GERDE. As a result of histological examination through phloxine-tartrazine staining, damage was observed on the apical surface of the esophagus. In the order of LPA, OMA, and GERDE, the injury site increased. (Fig. 1. Esophagus).

\section{ATV6V1B1 and GRPR regulation}

ATV6V1B1 positive reaction was increased by $458 \%$ in GERDE $(34,911 \pm 1,405 / 20,000,000$ pixel) compared to $\mathrm{Ctrl}(6,255 \pm 411 / 20,000,000$ pixel). The drug treatment group showed a lower increase than GERDE. Compared to Ctrl, OMA $(19,179 \pm 772 / 20,000,000$ pixel $)$ increased by 


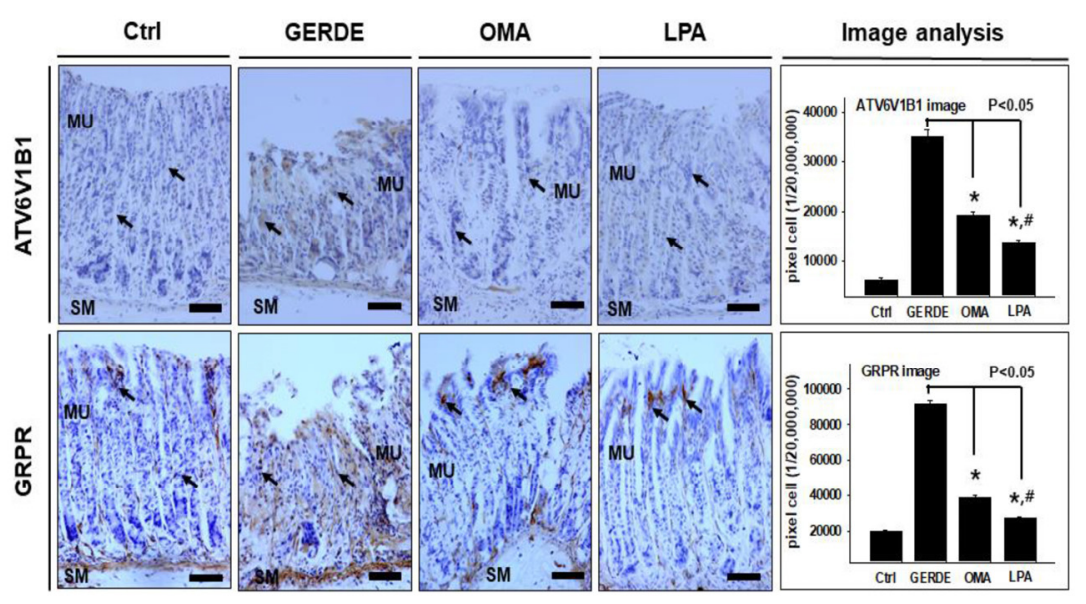

Fig. 2. The regulation of ATPase H+ Transporting V1 Subunit B1 (ATV6V1B1) and Gastrin-releasing peptide receptor (GRPR) in stomach by Omeprazole and Liriope platyphylla.

Immunohistochemical tests of ATP6V1B1 and GRPR were performed to investigate the acid secretion regulation effect of Liriope platyphylla. Both ATP6V1B1 and GRPR showed relatively low reaction patterns in the drug-treated groups, LPA and OMA, than in the GERDE group, and the LPA group showed a lower positive reaction than the OMA group. Image analysis was quantified using Image Pro and presented as means \pm standard error; * $p$ < 0.05 compared with GERDE; \#, $p$ < 0.05 compared with OMA; bar size, 50um. abbrevation same as Fig. 1.

$207 \%$ and LPA $(13,700 \pm 653 / 20,000,000$ pixel $)$ by $119 \%$. LPA had a $29 \%$ lower positive reaction than OMA (Fig. 2. ATV6V1B1).

GRPR positive reaction was increased by $361 \%$ in GERDE $(91,345 \pm 2,187 / 20,000,000$ pixel) compared to $\mathrm{Ctrl}(19,792 \pm 1,092$ /20,000,000 pixel). The drug treatment group showed a lower increase than GERDE. Compared to Ctrl, OMA $(38,869 \pm 1,513$ /20,000,000 pixel) increased by $96 \%$ and LPA $(27,387 \pm 989 / 20,000,000$ pixel) by $38 \%$. LPA had a $30 \%$ lower positive reaction than OMA (Fig. 2. GRPR).

\section{COX-1 inhibition}

COX-1 positive reaction was reduced by $71 \%$ in $\operatorname{GERDE}(21,637 \pm 1,117 / 20,000,000$ pixel $)$ compared to $\mathrm{Ctrl}(73,936 \pm 1,423 / 20,000,000$ pixel). The drug treatment group showed a lower decrease than GERDE. Compared to Ctrl, OMA (41,453 $\pm 1,585 / 20,000,000$ pixel) was reduced by $44 \%$, and LPA $(52,148 \pm 1,463 / 20,000,000$ pixel) was reduced by $29 \%$. LPA showed a $26 \%$ increase in positive reaction compared to OMA (Fig. 3.).

\section{8-OHdG regulation}

As a result of $8-\mathrm{OHdG}$ immunohistochemistry to investigate damage caused by oxidative stress on the injured site, $8-\mathrm{OHdG}$ positive reaction was increased by $323 \%$ in GERDE $(112,410 \pm 2,330$ /20,000,000 pixel) compared to $\mathrm{Ctrl}(26,593 \pm$ $1,284 / 20,000,000$ pixel). The drug treatment group showed a lower increase than GERDE. Compared to Ctrl, OMA $(58,412 \pm 1,663$ $/ 20,000,000$ pixel) increased by $120 \%$ and LPA 


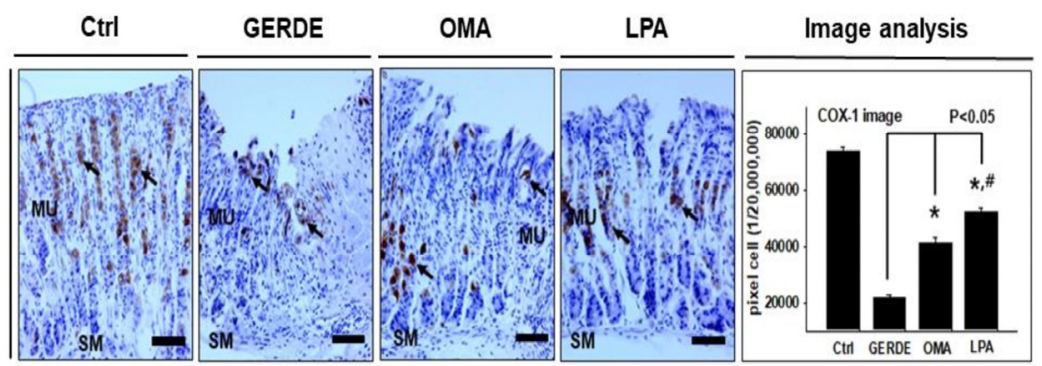

Fig. 3. The regulation of Cyclooxygenase-1 (COX-1) in stomach by Omeprazole and Liriope platyphylla.

Immunohistochemical test of COX-1 was performed to investigate the mucosal protective effect. The COX-1 positive reaction decreased in the GERDE, OMA, and LPA compared to the Ctrl, but the decrease was smaller in the LPA than in the GERDE and OMA. Image analysis was quantified using Image Pro and presented as means \pm standard error; * $p$ < 0.05 compared with GERDE; \#, $p<0.05$ compared with OMA; bar size, $50 \mu \mathrm{m}$. abbrevation same as Fig. 1.
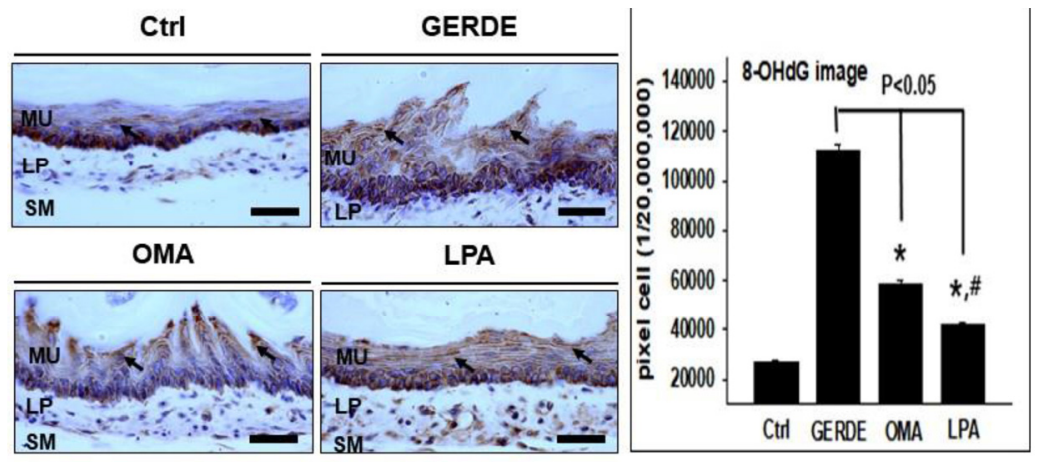

Fig. 4. The regulation of 8-hydroxy-2'-deoxyguanosine (8-OHdG) in esophagus by Omeprazole and Liriope platyphylla. Immunohistochemical test of $8-\mathrm{OHdG}$ was performed to investigate the antioxidant effects in the gastroesophageal junction and lower esophageal region. Compared to GERDE, the drug-treated group showed a lower reaction in the order of LPA and OMA. Image analysis was quantified using Image Pro and presented as means \pm standard error; *,$p<0.05$ compared with GERDE; \#, $p$ < 0.05 compared with OMA; bar size, 50 um. abbrevation same as Fig. 1.

$(41,790 \pm 1,229 / 20,000,000$ pixel $)$ by $57 \%$. LPA showed a $28 \%$ lower increase in positive reaction than OMA (Fig. 4.).

\section{5. cathelicidin regulation}

As a result of cathelicidin immunohistochemistry to investigate the formation of a protective barrier against the outside, cathelicidin positive reaction was reduced by $87 \%$ in GERDE $(4,815$ $\pm 281 / 20,000,000$ pixel) compared to $\mathrm{Ctrl}(36,755$ $\pm 716 / 20,000,000$ pixel). The drug treatment group showed a lower decrease than GERDE. Compared to Ctrl, OMA (11,364 \pm 519 $/ 20,000,000$ pixel) was reduced by $69 \%$ and LPA $(23,443 \pm 1,030 / 20,000,000$ pixel $)$ by $36 \%$. LPA showed a $106 \%$ higher increase in positive reaction than OMA (Fig. 5.).

\section{6. $p-J N K$ regulation}

As a result of $\mathrm{p}-\mathrm{JNK}$ immunohistochemistry to investigate the apoptosis modulating effect, the positive p-JNK reaction was increased by $695 \%$ 

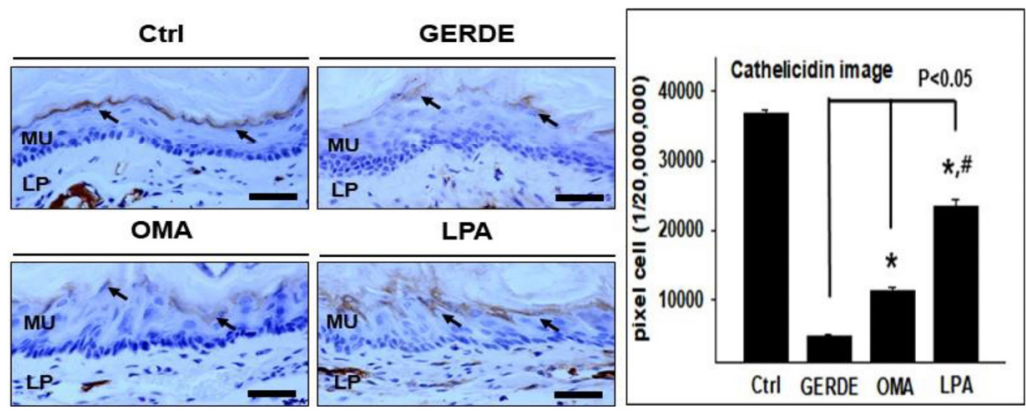

Fig. 5. The regulation of Cathelicidin in esophagus by Omeprazole and Liriope platyphylla.

Immunohistochemical test of Cathelicidin was performed to investigate the mucosal protective effect in the lower esophageal region. The positive reaction in GERDE was decreased compared to the Ctrl, and the LPA and OMA were less decreased compared to GERDE. In addition, the reduction of the positive reaction in LPA was smaller than that in OMA. Image analysis was quantified using Image Pro and presented as means \pm standard error; * $p$ < 0.05 compared with GERDE; \#, $p$ < 0.05 compared with OMA; bar size, 50um. abbrevation same as Fig. 1.
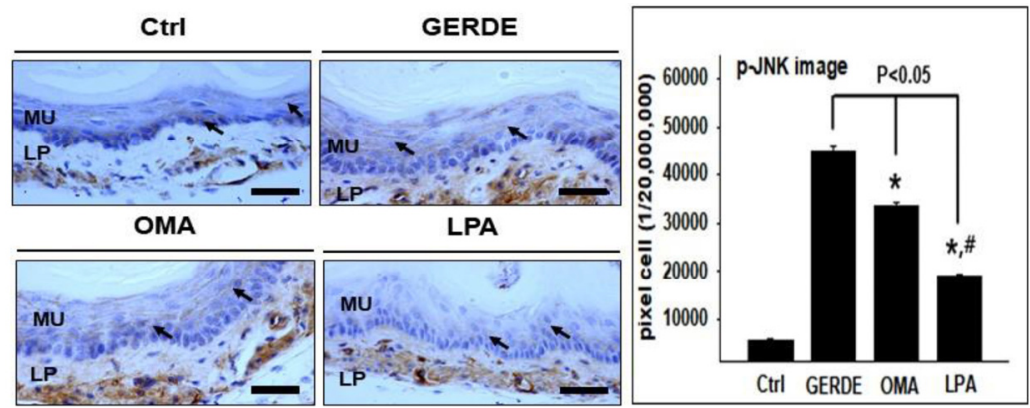

Fig. 6. The regulation of phospho c-Jun N-terminal kinase ( $\mathrm{p}-\mathrm{JNK}$ ) in esophagus by Omeprazole and Liriope platyphylla. Immunohistochemical test of $\mathrm{p}$-JNK was performed to investigate the effect of modulating apoptosis in the gastroesophageal junction and lower esophageal region. The positive reaction of $p-J N K$ was increased in the GERDE compared to Ctrl, and LPA and OMA increased less than GERDE. Image analysis was quantified using Image Pro and presented as means \pm standard error; *, $p<0.05$ compared with GERDE; \#, $p<0.05$ compared with OMA; bar size, 50um. abbrevation same as Fig. 1.

in GERDE $(44,709 \pm 1,005 / 20,000,000$ pixel $)$ compared to $\mathrm{Ctrl}(5,627 \pm 317 / 20,000,000$ pixel). The drug treatment group showed a lower increase than GERDE. Compared to Ctrl, OMA $(33,322 \pm 906 / 20,000,000$ pixel $)$ increased by $492 \%$ and LPA $(18,948 \pm 504 / 20,000,000$ pixel $)$ by $237 \%$. LPA showed a $43 \%$ lower increase in positive reaction than OMA (Fig. 6.).

\section{Discussion}

This study investigated the inhibitory effect of Liriope platyphylla on the onset of reflux esophagitis in the gastroesophageal junction and lower esophageal region using an animal model of reflux esophagitis. The effect of inhibiting damage to the esophageal mucosa was visually observed, and antioxidant, anti-inflammatory, gastric acid secretion control, mucosal protection, 
and apoptosis control were observed and compared with Omeprazole. Through this, it was confirmed whether Liriope platyphylla had a superior effect compared to the conventional treatment for GERD and reflux esophagitis.

First, through gross observation at the gastroesophageal junction and lower esophageal region and histological examination through phloxine-tartrazine staining, the GERDE-induced group showed severe hemorrhagic erosion, whereas the drug treatment group showed a low degree of damage in the order of LPA and OMA.

In this study, immunohistochemical tests of ATP6V1B1 and GRPR were performed to investigate the acid secretion regulation effect of Liriope platyphylla. ATP6V1B1 is a gene that transcribes the B1 subunit of the proton pump, and is an important factor involved in maintaining acid secretion homeostasis in the body ${ }^{25,26)}$. GRPR is a receptor for gastrin-releasing peptide (GRP), and when GRP is released from the enteric nervous system, the local concentration of gastrin increases to promote gastric acid secretion, and is closely related to inflammatory conditions $^{27,28)}$. Both ATP6V1B1 and GRPR showed relatively low reaction patterns in the drug-treated groups, LPA and OMA, than in the GERDE group, and the LPA group showed a lower positive reaction than the OMA group. These results indicate that Liriope platyphylla can inhibit acid secretion by regulating the proton pump function and gastrin secretion function, and furthermore, it shows the possibility that Liriope platyphylla can be used for various diseases caused by excessive gastric acid secretion as well as GERD.
The mucosal protective effect at the gastroesophageal junction was observed through COX-1 immunohistochemistry. COX-1 is an important enzyme in the biosynthesis process from arachidonic acid to prostaglandin, and promotes the formation of the lining of the gastric mucosa to protect the stomach and inhibits the secretion of gastric acid and pepsin ${ }^{29}$. Therefore, COX-1 can be said to be a representative gastric mucosal protective factor, and when COX-1 is deficient, gastric mucosal damage occurs primarily ${ }^{30}$. The COX-1 positive reaction decreased in the GERDE, OMA, and LPA compared to the Ctrl, but the decrease was smaller in the LPA than in the GERDE and OMA. The smaller decrease in positive reaction in the LPA indicates the possibility that Liriope platyphylla may have a direct effect on gastric mucosal protection and damage prevention.

Antioxidant effects in the gastroesophageal junction and lower esophageal region were observed by $8-\mathrm{OHdG}$ immunohistochemistry. $8-\mathrm{OHdG}$ is formed when reactive oxygen species (NOS) attack guanine at the dinucleotide site of somatic cells, and is an important biomarker indicating the degree of oxidative stress damage $^{31)}$. In general, an increase in $8-\mathrm{OHdG}$ not only means that the degree of oxidative damage has increased, but it is also widely used to measure risk factors for various diseases, including cancer $^{32)}$. As a result of the test, compared to GERDE, the drug-treated group showed a lower reaction in the order of LPA and OMA. This means that Liriope platyphylla has an antioxidant effect by inhibiting damage caused by free radicals, and that its effect may be superior 
to that of Omeprazole.

The mucosal protective effect in the lower esophageal region was observed by cathelicidin immunohistochemistry. Cathelicidin is an antibacterial peptide and plays an important role in human immunity by forming a protective barrier against external stimuli on the mucosal surface of the gastrointestinal tract ${ }^{33,34)}$. As a result of the study, the positive reaction in the GERDE group was decreased compared to the Ctrl group, and the LPA group and OMA group were less decreased compared to the GERDE group. In addition, the reduction of the positive reaction in the LPA group was smaller than that in the OMA group, demonstrating that Liriope platyphylla had a mucosal protective effect by promoting the formation of the gastric mucosal lining, enhancing the gastric mucosal protective effect, and improving the immune capacity of the mucosal surface.

The effect of modulating apoptosis in the gastroesophageal junction and lower esophageal region was observed by $\mathrm{p}-\mathrm{JNK}$ immunohistochemistry. P-JNK is a phosphorylated JNK, associated with an increase in apoptosis, is involved in cell apoptosis and inflammatory reactions in reaction to stress stimuli ${ }^{35-37)}$. In this study, the positive reaction of $p$-JNK was increased in the GERDE group compared to the Ctrl group, and the LPA group and OMA group increased less than the GERDE group. Therefore, it is shown that Liriope platyphylla is superior to Omeprazole in the degree of apoptosis control effect and it is thought that Liriope platyphylla was involved in signaling and mediating processes for apoptosis, thus inhibiting apoptosis.
As such, at the gastroesophageal junction and lower esophageal region, Liriope platyphylla showed more significant results than Omeprazole in inhibiting damage to the esophageal mucosa, controlling antioxidant, acid secretion, anti -inflammatory, mucosal protection, and controlling apoptosis. Based on these research results, it is expected that Liriope platyphylla will have a sufficiently high potential for use in promoting the formation of the gastric mucosa lining, inhibiting gastric mucosal damage caused by gastric acid, and inhibiting inflammation and apoptosis. Research is needed to explain the mechanisms for GERD treatment and prevention of Liriope platyphylla extracts in the future, and further research on safety and validation of Liriope platyphylla is thought to be necessary.

\section{Conclusion}

In this study, the effects of Liriope platyphylla on esophageal mucosa damage defense, acid secretion regulation, antioxidant, anti-inflammatory, mucosal protection, and apoptosis regulation were confirmed through reflux esophagitis animal model experiments.

1. Gross observation and histological examination confirmed the damage defense effect of the esophageal mucosa of Liriope platyphyla.

2. ATP6V1B1 and GRPR immunohistochemical tests confirmed the acid secretion regulation effect of Liriope platyphyla.

3. The protective effect of Liriope platyphyla on gastric mucosa was confirmed through COX-1 immunohistochemistry.

4. The antioxidant effect of Liriope platyphyla was 
confirmed through 8-OHdG immunohistochemistry.

5. The protective effect of Liriope platyphyla on the lower esophageal mucosa was confirmed by cathelicidin immunohistochemistry.

6. The apoptosis-regulating effect of Liriope platyphyla was confirmed through $\mathrm{p}-\mathrm{JNK}$ immunohistochemistry.

\section{Acknowledgement}

This study was supported by the clinical research fund of Pusan National University Hospital in 2021.

\section{References}

1. Jung, H. K., Hong, S. J., Jo, Y. J., Jeon, S. W., Cho, Y. K., Lee, K. J., et al. (2012). Updated guidelines 2012 for gastroesophageal reflux disease. Korean J Gastroenterol, 60(4), 195-218. https://doi.org/10.4166/kjg.2012.60.4.195

2. Fauci, A. S., Braunwald, E., Kasper, D. L., Hauser, S. L., Longo, D. L., Jameson, J. L., et al (2010). Harrison's principles of internal medicine (17th ed.). MIP. https://doi.org/10. 1111/j.1445-5994.2008.01837.x

3. Maton, P. N. (1991). Omeprazole. N Engl J Med, 324(14), 965-75. https://doi.org/10.1056/ NEJM199104043241406

4. Cheungpasitporn, W., Thongprayoon, C., Kittanamongkolchai, W., Srivali, N., Edmonds, P. J., Ungprasert, P., et al. (2015). Proton pump inhibitors linked to hypomagnesemia: A systematic review and meta-analysis of observational studies. Ren Fail, 37(7), 123741. https://doi.org/10.3109/0886022X.2015.1057800
5. Park, H. J., Park, S. H., Shim, K. N., Kim, Y. S., Kim, H. Y., Han, J. P., et al. (2016). The prevalence and clinical features of non-responsive gastroesophageal reflux disease to practical proton pump inhibitor dose in Korea: A multicenter study. Korean J Gastroenterol, 68(1), 16-22. https://doi.org/10. 4166/kjg.2016.68.1.16

6. Makunts, T., Alpatty, S., Lee, K. C., Atayee, R. S., \& Abagyan, R. (2019). Proton-pump inhibitor use is associated with a broad spectrum of neurological adverse events including impaired hearing, vision, and memory. Sci Rep, 9(1), 17280. https://doi.org/ 10.1038/s41598-019-53622-3

7. Kim, D. H., Lee, J. E., You, S. H., Lee, H. A., Park, J. H., Jung, J. H., et al. Proton pump inhibitor use and risk of osteoporotic fracture in Korean adults with peptic ulcer disease and gastroesophageal reflux disease. Seoul: National Evidence-based Healthcare Collaborating Agency. 2017: 67-70.

8. Roh, S. S., Choi, H. J., Kim, D. H., \& Seo, Y. B. (2008). Studies of anti-inflammation of Liriopis Tuber to autoimmunune diabetes in NOD mice. JPPKM, 22(4), 766-70.

9. Lee, E. S., Yang, S. Y., Kim, M. H., NamGung, \& U., Park, Y. C. (2011). Effects of root of Liriope Spicata on LPS-induced Lung Injury. JPPKM, 25(4), 641-9.

10. Im, J. G., Kang, M. S., Park, I. K., \& Kim, S. D. (2005). Dietary effect of Liriopis Tuber water extracts on the level of blood glucose and serum cholesterol in streptozotocin-induced diabetic rat. J East Asian Soc Dietary Life, 15(1), 20-8. 
11. Lee, I. J., \& An, J. Y. (2003). Hepatoprotective effects of water extract of Liriopis Tuber on carbon tetrachloride-induced hepatotoxicity in rats. Kor J Pharmacogn, 34(2), 166-71.

12. Lee, I. J., Baek, N. I., Cho, S. J., Bang, M. H., Kim, M. S., Park, C. G., et al. (1998). Cytotoxicity of steroid-saponins from the tuber of Liriope platyphylla W. T. Kor J Soc Agric Chem Biotechnol, 41(5), 390-4.

13. Jang, M. W., \& Lim, S. W. (2013). Experimental study for effect of Banhasasim-tang on mice with reflux esophagitis. Korean J Orient Int Med, 34(4), 362-3.

14. Lee, S. K., \& Lim, S. W. (2016). The administration of Jeungmiyijin-tang to rats with induced gastro reflux esophagitis. J Int Korean Med, 37(6), 1030-41. https://doi.org/ 10.22246/jikm.2016.37.6.103

15. Lee, S. T., \& Kwak, M. A. (2013). Effects of individual herbal components of Yijin-tang -gamibang in the rat reflux esophagitis. Korean J Orient Int Med, 34(2), 165-77.

16. Shin, M. K., Kim, E. S., Kim, T. R., Lim, H. C., \& Lee, Y. S. (2016). Effects of Jwa kum-whan on reflux esophagitis in rats. J Int Korean Med, 37(3), 495-507.

17. Kim, S. H., Roh, S. S., Lee, J. A., Shin, M. R., Lee, A. R., Koo, J. S., et al. (2019). Improving effects on rats with chronic acid reflux esophagitis treated of Coptidis Rhizoma extract. Kor J Herbol, 34(1), 117-24. https://doi.org/10.6116/kjh.2019.34.1.117

18. Lee, J. Y., Seo, B. I., \& Roh, S. S. (2016). Improving effect of Artemisiae Capillaris herba extract in reflux esophagitis rats. Kor $\mathbf{J}$ Herbol, 31(6), 37-44. https://doi.org/10.6116/ kjh.2016.31.6.37

19. Lee, J. A., Park, H. J., Kim, S. H., Kim, M. J., Kim, K. J., Shin, M. R., et al. (2019). Evaluation of Evodiae Fructus extract on the chronic acid reflux esophagitis in rats. Kor $\mathrm{J}$ Herbol, 34(2), 15-23. https://doi.org/10.15616/ BSL.2021.27.2.77

20. Kim, D. J., \& Roh, S. S. (2012). Effect on acute reflux esophagitis by Evodiae Fructus Aquous extract. Kor J Herbol, 27(1), 51-8. https://doi.org/10.6116/kjh.2012.27.1.51

21. Kwon, O. J., Lee, A. R., \& Roh, S. S. (2016). Improving effects on rats with reflux esophagitis treated with combined extract of young persimmon fruit and Citrus peel. Kor J Herbol, 31(1), 25-31. https://doi.org/10.6116/kjh.2016. 31.1 .25

22. Shin, M. H., Kim, E. S., \& Lee, Y. S. (2016). Suppressive effects of Ulmi Pumilae Cortex extracts on the reflux esophagitis in rat. JPPKM, 30(4), 257-65. https://doi.org/10. 15188/kjopp.2016.08.30.4.257

23. Shapiro, M., Green, C., Bautista, J. M., Dekel, R., Risner-Adler, S., Whitacre, R., et al. (2007). Assessment of dietary nutrients that influence perception of intra-oesophageal acid reflux events in patients with gastro -oesophageal reflux disease. Aliment Pharmacol Ther, 25(1), 93-101. https://doi.org/10.1111/ j.1365-2036.2006.03170.x

24. Nakamura, K., Ozawa, Y., Furuta, Y., \& Miyazaki, H. (1982). Effects of sodiumpolyacrylate (PANa) on acute esophagitis by gastric juice in rats. Jpn J Pharmacol, 32(3), 445-56. https://doi.org/10.1254/jjp.32.445

25. Stover, E. H., Borthwick, K. J., Bavalia, C., 
Eady, N., Fritz, D. M., Rungroj, N., et al. (2002). Novel ATP6V1B1 and ATP6V0A4 mutations in autosomal recessive distal renal tubular acidosis with new evidence for hearing loss. J Med Genet, 39(11), 796-803. https://doi.org/10.1136/jmg.39.11.796

26. Finberg, K. E., Wagner, C. A., Bailey, M. A., Păunescu, T. G., Breton, S., Brown, D., et al. (2005). The B1-subunit of the ATPase is required for maximal urinary acidification. PNAS, 102(38), 13616-21. https://doi.org/ 10.1073/pnas.0506769102

27. Czepielewski, R. S., Porto, B. N., Rizzo, L. B., Roesler, R., Abujamra, A. L., Pinto, L. G., et al. (2012). Gastrin-releasing peptide receptor (GRPR) mediates chemotaxis in neutrophils. PNAS, 109(2), 547-52. https://doi.org/ 10.1073/pnas.1110996109.

28. Petronilho, F., Danielski, L. G., Roesler, R., Schwartsmann, G., \& Dal-Pizzol, F. (2013). Gastrin-releasing peptide as a molecular target for inflammatory diseases: an update. Inflamm Allergy Drug Targets, 12(3), 172-7. https://doi.org/10.2174/1871528111312030003

29. Laine, L., Takeuchi, K., \& Tarnawski, A. (2008). Gastric mucosal defense and cytoprotection: bench to bedside. Gastroenterology, 135(1), 41-60. https://doi.org/10.1053/j.gastro.2008.05. 030

30. Cryer, B., \& Dubois, A. (1998). The advent of highly selective inhibitors of cyclooxygenase-a review. Prostaglandins Other Lipid Mediat, 56(5-6), 341-61. https://doi.org/10.1016/s0090 $-6980(98) 00064-1$

31. Zhou, X., Zhuang, Z., Wang, W., He, L., Wu, H., Cao, Y., et al. (2016). OGG1 is essential in oxidative stress induced DNA demethylation. Cell Signal, 28(9), 1163-71. https://doi.org/10.1016/j.cellsig.2016.05.021

32. Valavanidis, A., Vlachogianni, T., \& Fiotakis, C. (2009). 8-hydroxy-2' -deoxyguanosine (8-OHdG): a critical biomarker of oxidative stress and carcinogenesis. $\mathrm{J}$ Environ Sci Health C Environ Carcinog Ecotoxicol Rev, 27(2), 120-39. https://doi.org/10.1080/105905 00902885684

33. Sorenseon, O., Cowland, J. B., Askaa, J., \& Borregaard, N. (1997). An ELISA for hCAP-18, the cathelicidin present in human neutrophils and plasma. J Immunol Methods, 206(1-2), 53-9. https://doi.org/10.1016/S0022 -1759(97)00084-7

34. Hase, K., Eckmann, L., Leopard, J. D., Varki, N., \& Kagnoff, M. F. (2002). Cell differentiation is a key determinant of cathelicidin LL-37/human cationic antimicrobial protein 18 expression by human colon epithelium. Infect Immun, 70(2), 953-63. https://doi.org/10.1128/IAI.70.2.953-963.2002

35. Ip, Y. T., \& Davis, R. J. (1998). Signal transduction by the c-Jun N-terminal kinase (JNK) - from inflammation to development. Curr Opin Cell Biol, 10(2), 205-19. https://doi.org/10.1016/s0955-0674(98)80143-9

36. Choi, B. H., Hur, E. M., Lee, J. H., Jun, D. J., \& Kim, K. T. (2005). Protein kinase C delta-mediated proteasomal degradation of MAP kinase phosphatase-1 contributes to glutamate-induced neuronal cell death. J. Cell Sci, 119(7), 1329-40. https://doi.org/10. 1242/jcs.02837

37. Oh, H. L., Seok, J. Y., Kwon, C. H., Kang, 
S. K., \& Kim, Y. K. (2006). Role of MAPK in ceramide-induced cell death in primary cultured astrocytes from mouse embryonic brain. NeuroToxicology, 27(1), 31-8. https://doi.org/10.1016/j.neuro.2005.05.008

\section{ORCID}

Sang Hyun Ahn https://orcid.org/0000-0002-1526-4397

Il Shin Choi https://orcid.org/0000-0002-4005-683X

Ki Bong Kim https://orcid.org/0000-0002-0096-5836 